\title{
MATHEMATICAL MODELLING AND SIMULATION OF WIND TURBINE GENERATOR
}

\author{
Yi-tao DING* \\ School of Science, Xijing University, Shaanxi Xi'an 710123, China \\ E-mail: yitaoding1928@126.com
}

\begin{abstract}
This paper studied the mathematical model and calculation of each component of small and medium-sized wind turbine generators to improve its stability and power generation efficiency. The optimal modal gain and maximum power tracking range are obtained based on the traditional torque curve method. A complete maximum power tracking control strategy is developed based on the optimal torque curve method to conduct in-depth study and improvement. Finally, the feasibility and superiority of maximum power tracking control is verified via simulation. The improved maximum power tracking control can effectively accelerate the maximum power tracking process, making better capture of wind energy by the generator.
\end{abstract}

Keywords: Mathematical modelling, Simulation, Wind turbine generator.

\section{Introduction}

With the depletion of traditional energy sources such as coal and oil, wind energy has great potential as a renewable energy resource with less environmental pollution and huge reserves. Wind energy has been one of the fastest growing sources of energy in the world, with an annual increase of more than $30 \%$ in installed capacity as a result of reduction of utilization costs, short construction period of wind farm facilities and small area of land (Liu et al., 2015).

It is estimated that by 2020 , the installed capacity of wind power in the world will reach 1.231 billion $\mathrm{kW}$ and wind power will account for $12 \%$ of the global power generation (Boutoubat et al., 2013).

Since the beginning of the 21st century, the distributed energy grid-connected power generation market has been expanding day by day, which has led to the rapid development of small and medium sized wind turbine generators between $20 \mathrm{~kW}$ and $100 \mathrm{~kW}$. As of $2014,82 \%$ of small and medium sized wind turbine generators in the global market are grid connected (Janina et al., 2014). However, most small and medium sized wind turbine generators on the market today have problems such as simple structure, no pitch and yaw system, and single control method.

Simulation technology has been widely used for research and test of wind turbine generators for its advantages of being free from meteorological conditions and low investment.
As early as the beginning of the decade, foreign scholars began to study wind turbine performance using simulation methods (Zhao et al., 2013). At present, simulation technology has been applied in the design, manufacture, performance testing and research of wind power critical equipment and control systems, as well as analysis of wind turbine generator and wind farm operation (Can et al., 2015).

The simulation technology has been applied instead of the traditional testing using concrete equipment and other means. The research direction of simulation technology in wind power generation: Wind Energy Characteristics, Wind Farm Simulation, Simulation of Control System, Wind Turbine Simulation and Simulation Model (Yi et al., 2014).

\section{Modeling of Wind Power System 2.1 Mathematical drivetrain model of direct- driven wind turbine generator}

The drivetrain model of PMDC wind turbine generators is simple without low-speed shaft, highspeed shaft and gearbox in DFIGs (Thongam et al., 2011). The simple mass model assumes a drivetrain spindle as a rigid axis. Its motion equation can be expressed as:

$$
\left\{\begin{array}{c}
\mathrm{I} \dot{\omega}_{r}=T_{m}-T_{\mathrm{e}}-B \omega_{r} \\
I=I_{r o t}+I_{g e n}
\end{array}\right.
$$


where $\hat{\omega}_{\mathrm{r}}$ denotes the rotor speed; $\mathrm{T}_{\mathrm{m}}$ denotes the mechanical torque; $\mathrm{T}_{e}$ denotes the electromagnetic torque; ${ }^{\mathrm{B}}$ denotes the damping coefficient; ${ }^{\mathrm{I}}$ denotes the equivalent rotating inertia; $\mathrm{I}_{\text {rot }}$ denotes the rotor rotating inertia; ${ }^{\mathrm{I} e n}$ denotes the generator rotating inertia.

\subsection{Mathematical model of permanent magnet synchronous generator}

The permanent magnet synchronous generator consists of a permanent magnet that forms the rotor magnetic field.

The torque is generated by the interaction between the rotor magnetic field and the rotating magnetic field generated by the stator current (Raza et al., 2013).

According to the knowledge of electromotor, the mathematical model of permanent magnet synchronous generator under the $d q$ synchronous rotation coordinate system is as follows:

$$
\left\{\begin{array}{c}
\frac{d i_{d}}{d t}=-\frac{R_{a}}{L_{d}} i_{d}+\omega_{e} \frac{L_{q}}{L_{d}} i_{q}+\frac{1}{L_{d}} u_{d} \\
\frac{d i_{q}}{d t}=-\frac{R_{a}}{L_{d}} i_{q}-\omega_{e}\left(\frac{L_{d}}{L_{q}} i_{d}+\frac{1}{L_{q}} \lambda_{0}\right)+\frac{1}{L_{q}} u_{q}
\end{array}\right.
$$

where $i_{d}$ and $i_{q}$ are the $d$ and $q$ axis currents of the generator respectively; $L_{d}$ and $L_{q}$ are the $d$ and $q$ axis inductance respectively; $R_{a}$ is the stator resistance; $\omega_{e}$ is the electrical angular frequency; $\lambda_{0}$ is the permanent magnet flux linkage; $u_{d}$ and $u_{q}$ are $d$ and $q$ axis components respectively.

If the $d$ and $q$ axis inductance of the generator is equal, the model is as follows:

$$
\left\{\begin{array}{c}
\frac{d i_{d}}{d t}=-\frac{R_{a}}{L_{d}} i_{d}+\omega_{a} i_{q}+\frac{1}{L} u_{d} \\
\frac{d i_{q}}{d t}=-\frac{R_{a}}{L_{d}} i_{q}-\omega_{e}\left(i_{d}+\frac{1}{L} \lambda_{0}\right)+\frac{1}{L} u_{q}
\end{array}\right.
$$
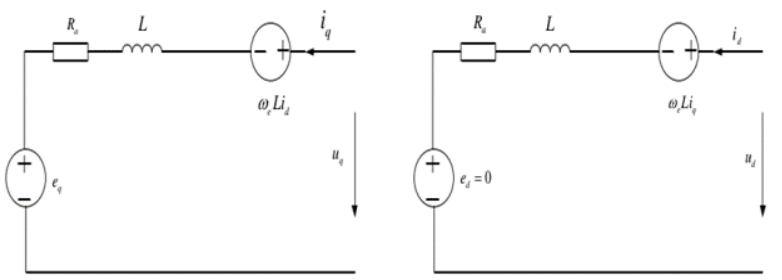

(a) Equivalent circuit of $q$ axis (b) Equivalent circuit of $d$ axis

Figure 1 Equivalent circuit of permanent magnet synchronous generator

Figure 1 shows the equivalent circuit of permanent magnet synchronous generator in the $d$ and $q$ synchronous rotating coordinate system by analyzing the mathematical model of the motor.

PMDC synchronous wind turbine generators are studied for the control. Through the adjustment of inverter control system, the generator can operate in the full apower range and the power generation efficiency is improved.

\section{Optimal Torque Control for Wind Turbine Generators \\ 3.1 Optimal torque control for traditional wind turbine generators}

The wind turbine power generation topology without considering grid-based converter is adopted. A simulation toolbox Simulink in Matlab is used to establish the maximum power tracking control system for wind turbine generators (Figure 2).

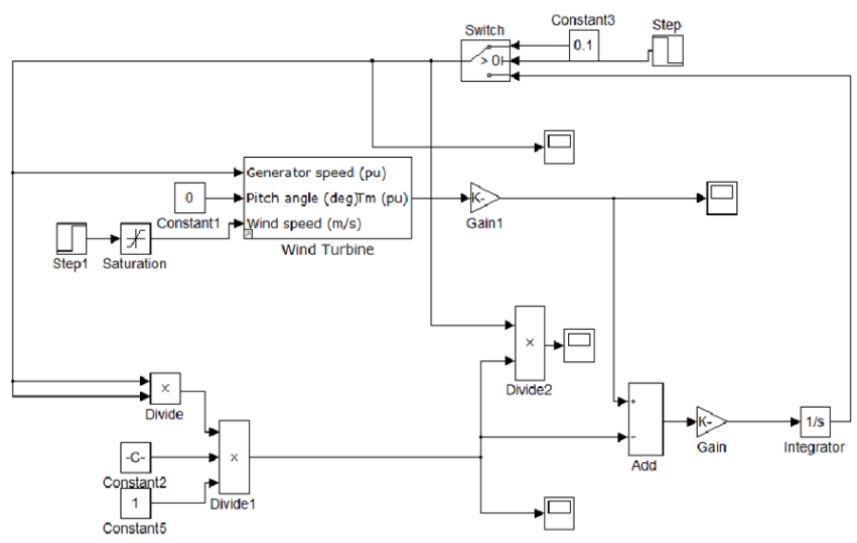

Figure. 2 The model of maximum power tracking control system 
Set wind speed at initial stable stage of the wind turbine generator of $5 \mathrm{~m} / \mathrm{s}$ and the step of $6 \mathrm{~m} / \mathrm{s}$ at $\mathrm{t}=$ 4 , get the power and speed curves of wind speed mutation (Figure 3). It can be seen that when the wind speed increased from $5 \mathrm{~m} / \mathrm{s}$ to $6 \mathrm{~m} / \mathrm{s}$, the speed and output power of wind turbine generator increased from $87.5 \mathrm{rpm}$ to $97.5 \mathrm{rpm}$ and from $3 \mathrm{~kW}$ to $5.3 \mathrm{~kW}$ respectively.
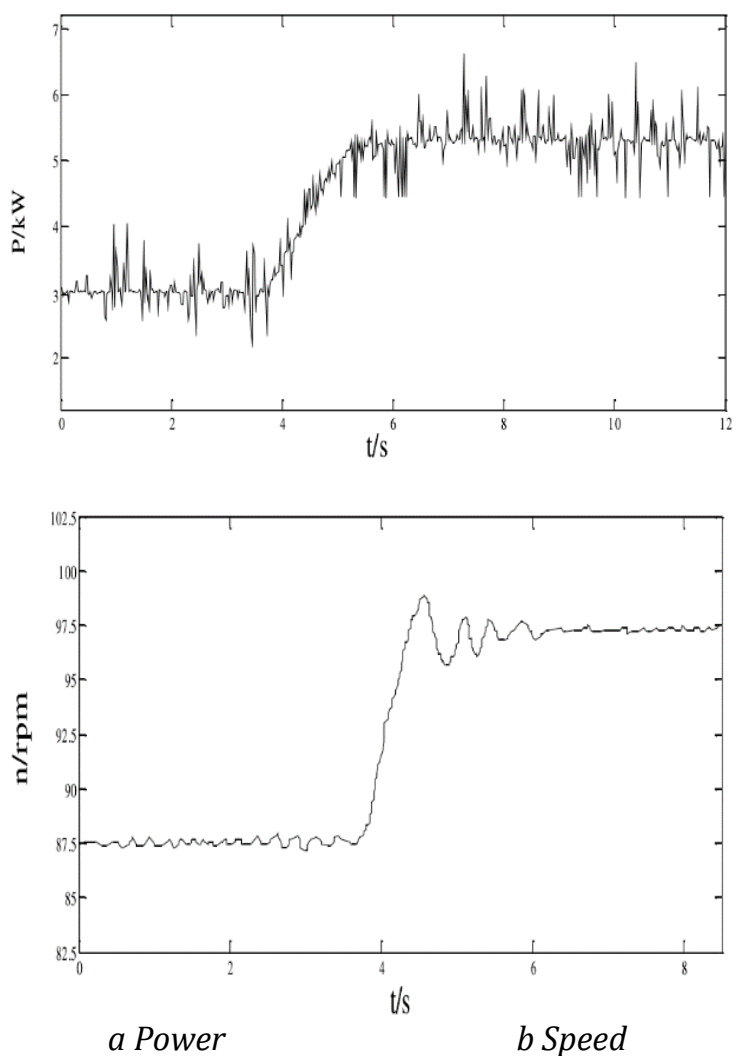

Figure. 3 The power and speed curves of wind speed mutation

It can be seen that, it lasted for about 2 seconds from the step change of wind speed to the optimum steady speed of the wind turbine benefiting from small rotating inertia of small and medium-sized wind turbine generators. The wind speed changes rapidly, and the torque and speed cannot change following the gain coefficient consistently, resulting in deviation, which leads to reduced maximum wind energy capture capacity of the generator and power generation.

Therefore, how to make the generator reach steady power quickly is the key to improving the control strategy.

\subsection{Improved optimal torque control}

To solve the above problem, this section calculates the optimal torque coefficient by genetic algorithm, and compensates for the given torque in the traditional optimal torque control. Figure 4 shows Schematic diagram of improved optimal torque control.

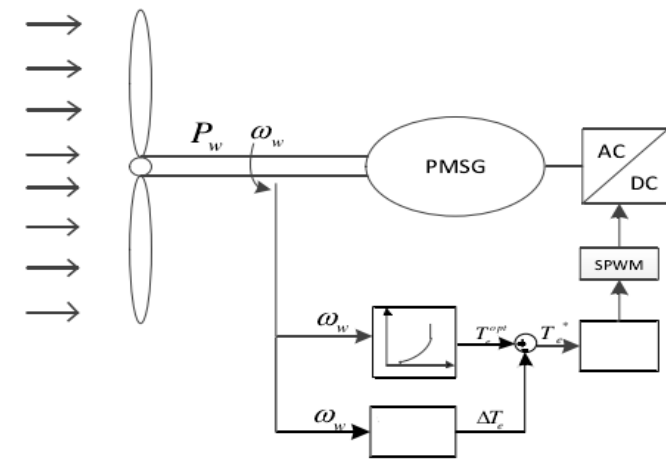

Figure. 4 Schematic diagram of optimal torque control

The principle is as follows: When the rotor speed change from $\omega 0$ to $\omega 1$, slight disturbance is added to the torque value at each feedback, becoming:

$$
\mathrm{T}_{\theta}^{*}=k_{\text {opt }} \omega_{1}^{2}+\Delta T
$$

where $k_{\text {opt }}$ is the optimal torque gain factor, which is determined by the wind turbine parameters.

If a proper $\Delta \mathrm{T}$ is chosen, the wind turbine generator quickly reaches the maximum power tracking point for torque control. The method is:

(1) Population initialization: When the speed of generator increases, ten points from T0 to TEopt is chosen for the given torque to form the initial population.

(2) Construction of fitness function: In this paper, the variable selected for the fitness function is the difference between the speed feedback obtained after each torque compensation and the speed feedback of the previous generation. This optimal torque control will make the generator reach the optimal speed steadily and fast.

Therefore, the constructed fitness function is the absolute speed deviation after each cycle.

(3) Selection operation: In this paper, the proportional selection method is used to calculate the individual fitness of each parent and the sum of fitness of all parents. The ratio of the two values corresponds to each individual. The individuals with higher fitness are more likely to be chosen.

(4) Recombination and mutation: In the first cycle, the mutation rate is set larger, and then gradually reduced, helping to capture the maximum power point.

(5) A new generation of population: After the above process is completed, a new generation of population is generated and returned to the second section, until the speed mutation is eliminated and the generator operates stably.

During the simulation analysis, we also set wind speed at initial stable stage of the wind turbine generator of $5 \mathrm{~m} / \mathrm{s}$ and the step of $6 \mathrm{~m} / \mathrm{s}$ at $t=4$, get the power and speed curves of wind speed mutation (Figure 5). It can be seen that when the wind speed increased from $5 \mathrm{~m} / \mathrm{s}$ to $6 \mathrm{~m} / \mathrm{s}$, the speed and output power of wind turbine generator increased from $87.5 \mathrm{rpm}$ to $97.5 \mathrm{rpm}$ and from $3 \mathrm{~kW}$ to $5.3 \mathrm{~kW}$ 
respectively. This process is obviously fast, taking about 1 s to restore steady state.
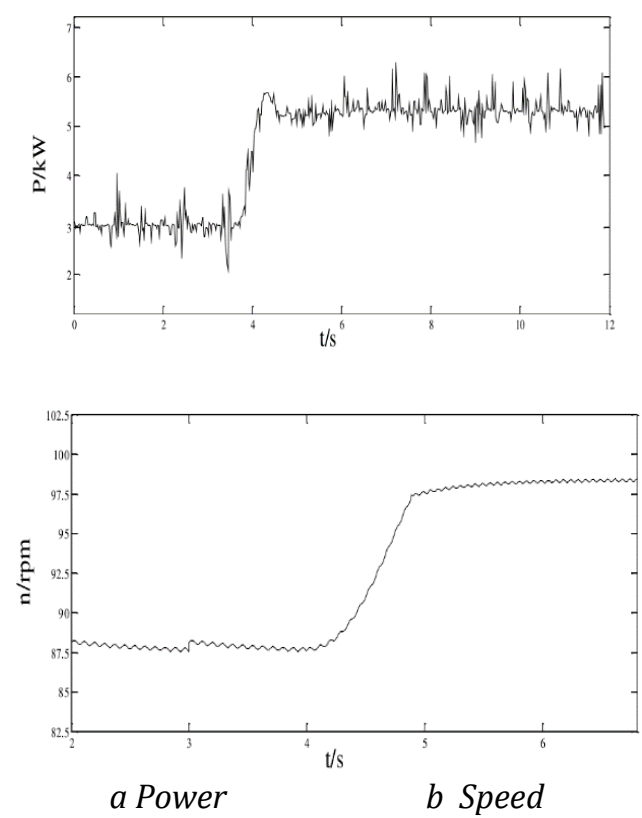

Figure. 5 The power and speed curves of wind speed mutation

\subsection{Comparative analysis}

The traditional optimal torque control and the improved optimal torque control were simulated respectively to verify the superiority of the latter. When the wind speed changed from $5 \mathrm{~m} / \mathrm{s}$ to $6 \mathrm{~m} / \mathrm{s}$, observe the process in two control methods, the power change is shown through comparison (Figure 6).

The red curve is the traditional control method and the blue curve is the improved control method. It can be seen that, when the wind speed changes, it takes about $2 \mathrm{~s}$ to restore the maximum power point in the traditional method, which is obviously longer and the tracking speed is obviously slower. The improved control method can greatly improve the dynamic performance of generator. Under the same conditions, it took only about $1 \mathrm{~s}$ to restore the maximum power point, indicating that the improved control method can effectively speed up the maximum power tracking process, making better capture of wind energy by the generator.

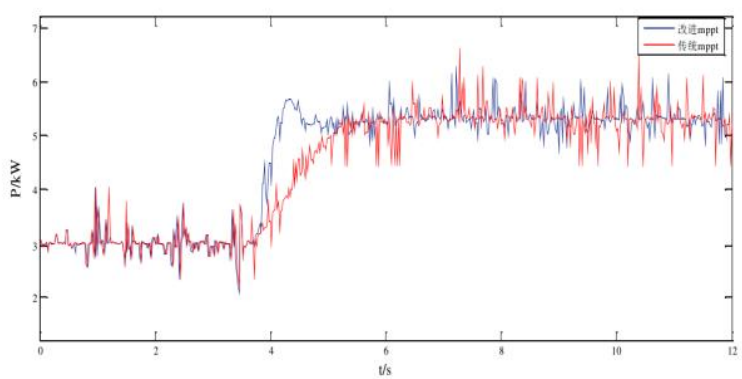

Figure. 6 Power changes of two different maximum power tracking control

\section{Conclusion}

In this paper, the mathematical model of each subsystem of small and medium-sized wind turbine generators is constructed by using mechanism modeling. The maximum wind energy capture control with wind speed mutation is studied.

The optimal torque gain coefficient is calculated to regulate the speed and torque for maximum power tracking. The feasibility and superiority of the improved maximum power tracking control are verified through simulation.

The results show that the improved maximum power tracking control can effectively accelerate the maximum power tracking process, making better capture of wind energy by the generator.

\section{References}

[1] Y.X. Liu, L.Z. Ren and Y.B. Li (2015). The industrial performance of wind power industry in China. Renewable and Sustainable Energy Reviews, vol.8, no.43, p.644-655.

[2] M. Boutoubat, L. Mokrani and M. Machmoum (2013). Control of a wind energy conversion system equipped by a DFIG for active power generation and power quality improvement. Renewable Energy, vol.6, no.32, p.378-386.

[3] C.K. Janina (2014). The impact of wind power generation on the electricity price in Germany. Energy Economics, vol.44, no.7, p.270-280.

[4] Z. Zhao, Y. Hong and Z. Jian (2013). A critical review of factors affecting the wind power generation industry in China. Renewable and Sustainable Energy Reviews, vol.6, no.19, p.499508.

[5]H. Can, L. Fangxing and J. Zhiqiang (2015). Maximum Power Point Tracking Strategy for Large-Scale Wind Generation Systems Considering Wind Turbine Dynamics. IEEE Transactions on Industrial Electronics, vol.4, no.62, p.2530-2539.

[6] L. Yi, S. Lin and T. Guangyu (2014). Optimal sizing and control strategy of isolated grid with wind power and energy storage system. Energy Conversion and Management, vol.24, no.6, p.407 415.

[7] J.S. Thongam and M. Ouhrouche (2011). MPPT control methods in wind energy conversion systems. Rijeka,Croatia: INTECH Open Access Publisher, vol.16, no.34, p.339-360.

[8] S.M. Raza, H. Goto and H J. Guo (2013). A novel algorithm for fast and efficient speed-sensorless maximum power point tracking in wind energy conversion systems. IEEE Transactions on Industrial Electronics, vol.58, no.1, p.29-36. 